\title{
Design Considerations for Oligo(p-phenyleneethynylene) Organic Radicals in Molecular Junctions
}

\author{
Martin Sebastian Zöllner*, Rukan Nasri*, Haitao Zhang, Carmen Herrmann** \\ * Shared first authors \\ ** Corresponding author: carmen.herrmann@chemie.uni-hamburg.de
}

April 21th 2020

\begin{abstract}
Spin polarization in the electron transmission of radicals is important for understanding singlemolecule conductance experiments focusing on shot noise, Kondo properties or magnetoresistance. We study how stable radical substituents can affect such spin polarization when attached to oligo(pphenyleneethynylene) (OPE) backbones. We find that it is not straightforward to translate the spin density on a stable radical substituent into spin-dependent transmission for the para-connected wires under study here, owing to increased steric interactions compared with meta-connected wires, and a resulting twisting of the radical substituent and OPE $\pi$ systems. The most promising example is a t-butyl nitroxide substituent, which, despite little pronounced spin delocalization onto the backbone, yields a spin-dependent transmission feature which one might be able to shift towards the Fermi energy by additional substituents. We also find that for bulkier substituents, dispersion interactions with the substituent can lead to twisting of one of the outer OPE rings, reducing the overall conductance. As a further potential design consideration, attaching radicals via linkers might increase the possibilities for spin-dependent intermolecular and molecule-electrode interactions.
\end{abstract}




\section{Introduction}

Electron transport through molecules is relevant for a variety of scientific fields, such as nanotechnology, biochemistry, catalysis, and materials science [1-3]. An important model system for understanding such transport processes are single-molecule junctions [4-10], which are often constructed via mechanical or electromigrated break-junction techniques (see Figure 1).

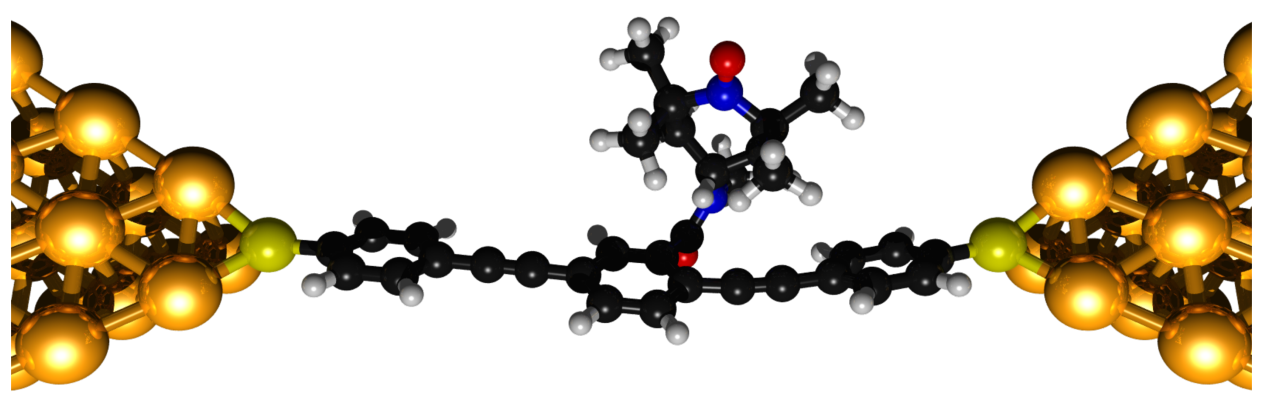

Figure 1: Illustration of a molecular bridge between two gold electrodes, forming a molecular junction. An OPE backbone with a TEMPO radical substituent is shown, as was studied experimentally with respect to its single-molecule magnetoresistance properties [11].

Beyond serving as a means to understand molecular function with certain applications in mind, single-molecule junctions also provide a highly appealing view into how molecules behave under unusual conditions, i.e., in nonequilibrium and possibly exposed to additional stimuli such as light, (static) electrical or magnetic fields, or mechanical control [12-19]. Molecules display particularly rich behavior when they feature unpaired spins, as present in many transition metal complexes and in organic radicals [20-30]. In the past few years, such molecules have been more and more in the focus of single-molecule break junction experiments with nonmagnetic electrodes, and in particular their response to magnetic fields [11,31], Kondo properties [32-35] and shot noise resulting from spin correlations $[36,37]$ have been studied. Radicals adsorbed on graphene were also found to enhance the conductance and the Seebeck coefficient of graphene nanoconstrictions based on first-principles simulations [38].

Magnetoresistance in organic molecules has been studied in crystals, thin films and related structures, and depending on the type of molecule and experimental setup, different mechanisms are discussed [39]. For single-molecule junctions, it is much less clear which physical mechanisms are responsible for the observed magnetoresistance behavior. Similarly, the link between Kondo signatures and chemical structures is not fully understood [40], while shot-noise measurements on spin-polarized sys- 
tems can be related to the difference between majority- and minority-spin transmission $[36,37,41,42]$. First principles simulations as provided by density functional theory (DFT) have proven valuable for understanding magneto-structural correlations in magnetic molecules [43,44], and are a promising means of gaining insight into structure-property relations for the above-mentioned experiments.

Interestingly, also molecules without any intrinsic magnetic moment but with a helical structure can display spin-dependent electron transport behavior, a phenomenon which has been named chiral induced spin selectivity [45-60] and which is commonly attributed to Rashba-type spin-orbit coupling. This, along with other observations such as anisotropic magnetoresistance [31,61,62] and electrodeand metal-center-dependent magnetoresistance [63,64] suggests that spin-orbit coupling, possibly resulting from interactions with the electrodes, may play a role in understanding single-molecule magnetoresistance. Given that spin-orbit coupling at metal-molecule interfaces is far from being understood, in particular from a first-principles perspective, we will focus here on those aspects of possible relevance for the above-mentioned experiment which can be described well by present-day first-principles methods, namely molecular structures, spin density distributions, and spin polarization of electron transmission.

Magnetoresistance has also been observed at room temperature in self-assembled monolayers which, at least formally, do not feature any unpaired spins $[65,66]$. This has been attributed to a (cooperative) interface effect. Since also in single-molecule conductance measurements, the molecule bridging between the electrodes is typically surrounded by other molecules of the same type due to the preparation process, intermolecular interactions and cooperative effects could also play a role in (formally) single-molecule experiments. It has also been suggested based on DFT calculations that the surface could play a noninnocent role in thiol adsorption on gold, possibly leading to spin polarization at the interface [67-71], which might interact with the spin on the molecule [68]. Also, a radical could loose its spin when attached to a metal surface [72], which can depend subtly on the details of the environment [73]. Further, there is debate in the literature as to whether thiols keep or loose their hydrogen atoms / protons when attaching to gold [74,75]. Overall, there are many open questions regarding interface-related effects leading to spin polarization, and the accurate first-principles description of molecule-metal interfaces in general is not trivial [40,76-85], which is why we focus in a first step on the purely molecule-related properties of single radical wires which keep their their spin when attached to electrodes.

We will study how attaching radical substituents to conjugated backbones can affect overall transmission and spin polarization, and how steric effects, dispersion interactions and structural flexibility 
play a role here. We focus on a monosubstituted oligo(p-phenyleneethynylene) (OPE) backbone, inspired by previous experimental and theoretical work on TEMPO-OPE [11].
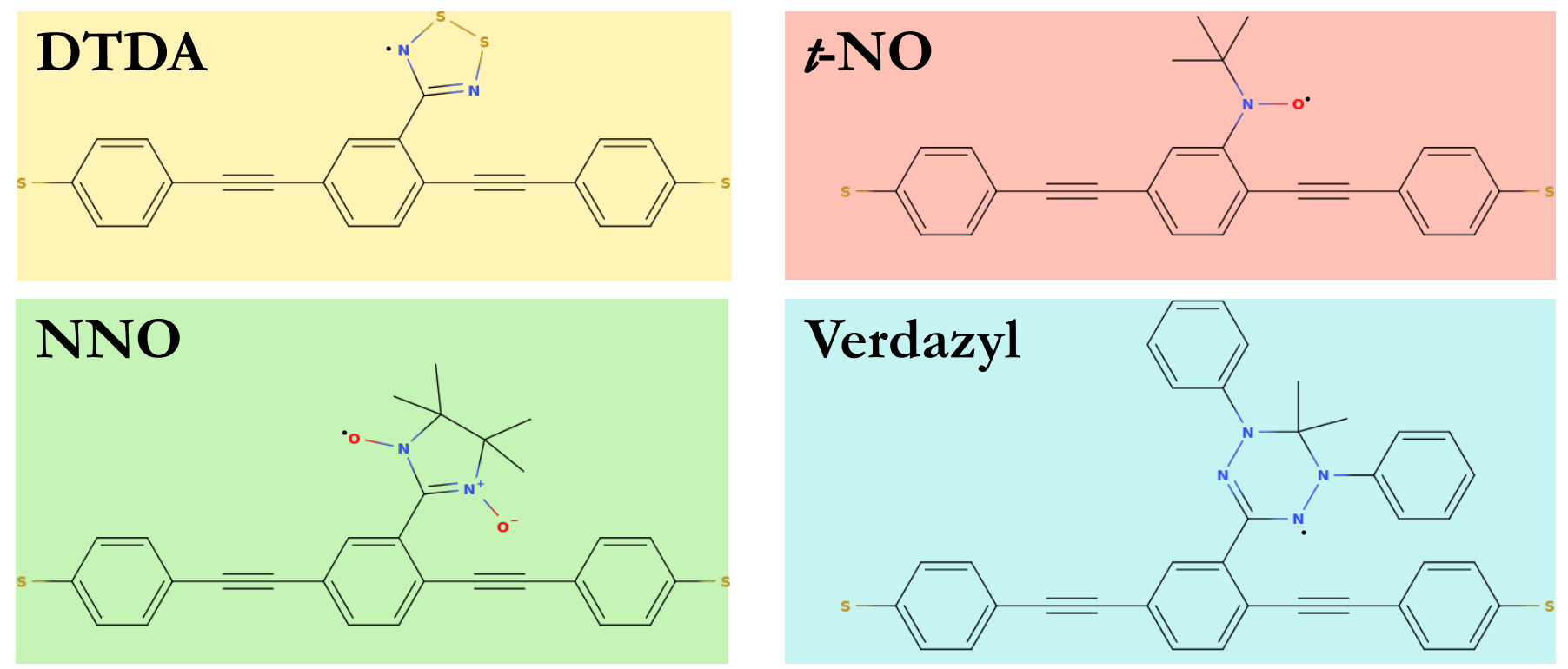

Figure 2: Lewis structures of the radical wires under study. A DTDA (a), a tert-butyl nitroxide (b), a nitronyl nitroxide (c), and a verdazyl (d) radical were attached to an OPE-backbone with thiolate linkers.

The conductance properties of substituted OPE backbones have been studied experimentally and theoretically before, in particular in the context of negative differential conductance [86,87] and modulating destructive quantum interference via controlling resonance structures $[88,89]$. The effect of radical substituents on OPE transmission has not been studied in detail yet. We choose four such substituents, 1,2,3,5-dithiadiazolyl (DTDA), nitronyl nitroxide (NNO), tert-butyl nitroxide $(t$ NO) and verdazyl radicals [90] (see Figure 2). They were chosen because they are relatively stable, leading to considerable potential as components of molecular materials with technologically relevant properties [91], e.g., DTDA is an important building block for solid-state organic conductors and magnets $[92,93]$. We have studied the $t$-NO substituent previously with respect to its potential for increasing exchange interactions between spin centers linked by an organic bridge [94]. Our DFT data suggested that the effect of $t$-NO is substantial, being half as large as for (unstable) idealized radical substituents such as $-\mathrm{O} \cdot$. Given common trends between spin coupling and conductance through molecular bridges [95,96,96-103], this could suggest that $t$-NO (and the other stable radical substituents studied here) could have a considerable effect on spin polarization in electron transport through OPE wires. We again compare with an artificial (unstable) radical substituent, and also 
discuss the potential importance of structural flexibility of the "original" TEMPO-OPE system as an outlook.

\section{How much spin polarization can we achieve by attaching stable radical substituents directly to OPE backbones?}

We are evaluating single-molecule conductance assuming coherent tunneling as the dominant transport mechanism (Landauer regime). While this assumption can break down for long wires, high temperatures and in electronic resonance [7,104], it is reasonable for a (substituted) OPE backbone with three phenyl units $[11,105,106]$. The zero-bias conductance $g$ can be estimated in this regime from the transmission at the Fermi energy $E_{F}$,

$$
g=\frac{e}{h}\left(T^{\uparrow}\left(E_{F}\right)+T^{\downarrow}\left(E_{F}\right)\right)
$$

where $e$ is the unit charge and $h$ Planck's constant, and $T^{\uparrow}$ and $T^{\downarrow}$ are the spin-up / majorityspin and spin-down / minority-spin transmission, respectively. For spin-polarized systems such as radicals, $T^{\uparrow}$ and $T^{\downarrow}$ can be different, which is referred to as spin polarization. We model $T^{\uparrow}$ and $T^{\downarrow}$ via a Green's function approach combined with density functional theory calculations for zero-bias electronic structures in the wide-band limit, as described, e.g., in Refs. [107-110] and as detailed in the Supporting Information.

Spin flips, which may play a role in tunneling processes [111-114], are neglected here. This appears justified by the good agreement between shot noise measurements and spin-flip-free theoretical descriptions for spin-polarized systems [41]. It is also supported by validation calculations on simple radicals, in which a two-component description allowing, in principle, for spin flips, did not lead to substantial changes of transmission functions for collinear spin arrangements, even when including spin-orbit coupling (see Supporting Information). 


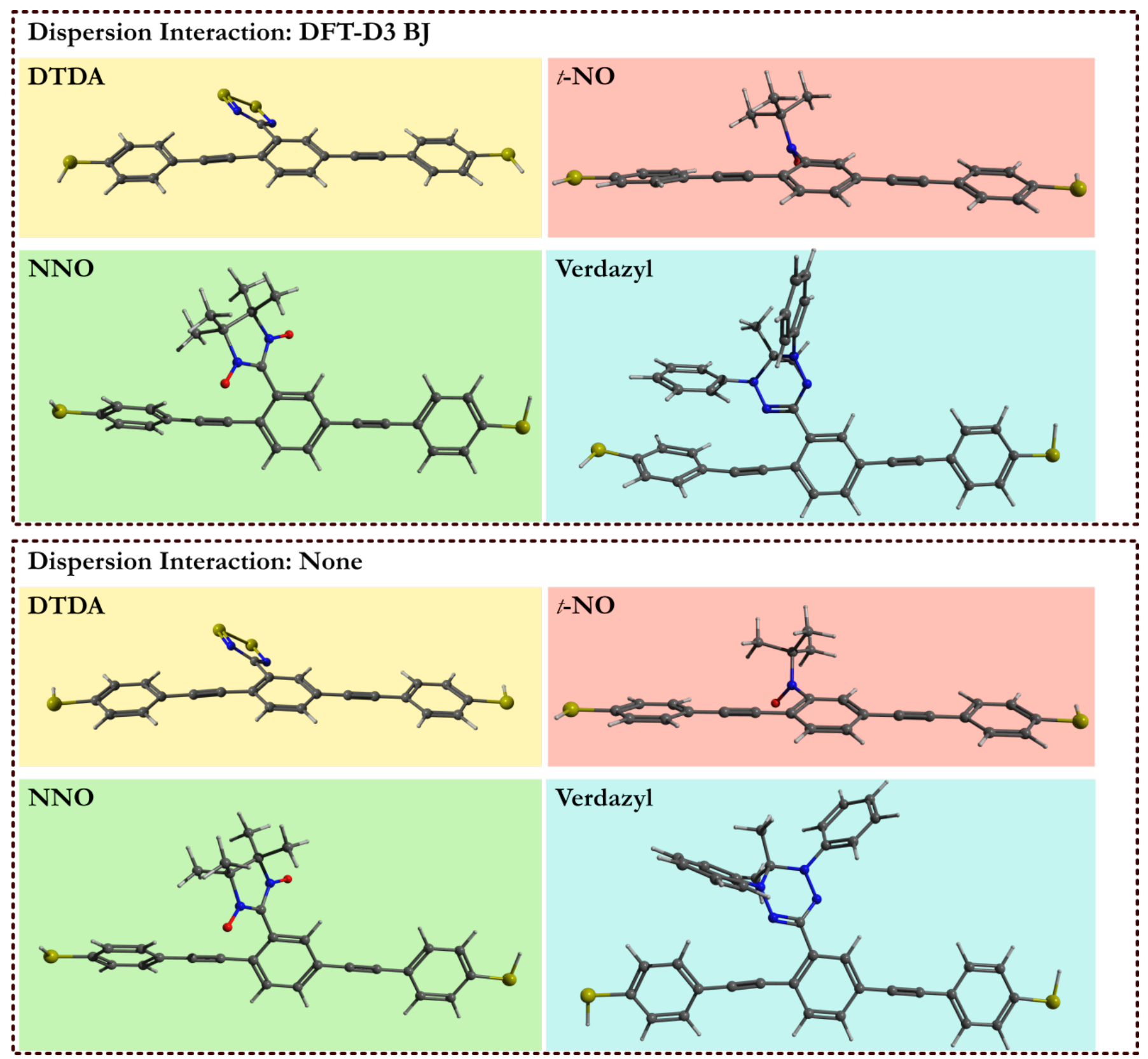

Figure 3: Optimized molecular structures of the isolated OPE derivatives functionalized with organic radical substituents $(\mathrm{DTDA}=1,2,3,5$-dithiadiazolyl, $t$-NO=tert-butyl nitroxide, $\mathrm{NNO}=$ nitronyl nitroxide). While for the DTDA and the $t$-NO radical the OPE backbone remains (nearly) planar, the presence of the NNO and verdazyl radicals leads to twisting of one of the phenyl rings.

As far as the molecular structures are concerned, depending on the organic radical, one of the outer OPE phenyl rings can be twisted (see Figure 3 and Table 1). This is in line with previous theoretical work suggesting that these rings can be easily rotated [115]. For the small organic residues (DTDA, $t$ NO), only minor distortions occur and the OPE backbone remains fully or nearly planar, respectively. For the more bulky radicals (NNO, verdazyl), the outer ring closest to the radical rotates (up to 63 
degrees for verdazyl), partially breaking $\pi$ conjugation in the conducting backbone. For the verdazyl radical, this structural change is mainly induced by dispersion interactions, as indicated by structure optimizations without empirical dispersion corrections showing much less twisting (see Figure 3). For the other radicals, the inclusion of dispersion corrections does not influence the OPE backbone significantly. A stronger twisted ring translates into a lower overall minimum of transmission (see Figure 4), in line with previous research [116]. For a summary of the transmissions at the Fermi energy for the different molecules under study, see Table 2.

Table 1: Dihedral angles for the optimized structures shown in Figure 3 (in degrees). Upper line of each entry: dihedral angles defining twisting between radical substituent and OPE backbone; lower line: dihedral angles defining twisting between central ring and twisted ring of the OPE backbone.

\begin{tabular}{l|l|l}
\hline \hline & with dispersion correction & no dispersion correction \\
\hline DTDA & $33.1 / 34.9$ & $32.6 / 34.5$ \\
& $0 / 0$ & $0 / 0$ \\
\hline$t$-NO & 69.0 & 65.1 \\
& $18.3 / 21.4$ & $15.5 / 16.5$ \\
\hline NNO & $47.0 / 51.0$ & $47.5 / 52.8$ \\
\hline verdazyl & $51.7 / 54.0$ & $46.3 / 48.4$ \\
\hline me-NO & $31.8 / 35.8$ & $25.7 / 32.9$ \\
& $52.1 / 61.0$ & $18.8 / 22.1$ \\
\hline TEMPO (cis) & 0.4 & 0.3 \\
\hline TEMPO (trans) & $0 / 0 . a$. & 0.1 \\
\hline \hline & $17.2 / 17.6$ & n.a. \\
\hline
\end{tabular}


Table 2: Calculated transmissions at the estimated Fermi energy $\left(E_{F}\right)$ of $-5 \mathrm{eV}$ using B3LYP/LANL2DZ for the organic radicals optimized with and without dispersion interaction. Additionally, the calculated transmissions at $E_{F}$ of the OPE, the methyl-nitroxide (me-NO) and both forms of the TEMPO-OPE radical are shown. Two factors influence the amount of transmission. A larger dihedral angle between the outer ring and the central ring of the OPE backbone leads to a lower transmission, and a shift of the transmission peak corresponding to the HOMO enhances the transmission at $E_{F}$, as seen for the verdazyl radical. For the me-NO radical, $T^{\uparrow \uparrow}$ and $T^{\downarrow \downarrow}$ differ significantly, and $T^{\text {tot }}$ is larger than for the other molecules due to the presence of an additional transport peak close to $E_{F}$ of the SOMO.

\begin{tabular}{l|cc}
\hline \hline Molecule & \multicolumn{2}{c}{ Transmission } \\
& $T^{\uparrow \uparrow}\left(E_{f}\right)$ & $T^{\downarrow \downarrow}\left(E_{f}\right)$ \\
\hline \multicolumn{3}{c}{ with dispersion } \\
\hline DTDA & 0.007 & 0.007 \\
$t$-NO & 0.006 & 0.006 \\
NNO & 0.003 & 0.003 \\
verdazyl & 0.004 & 0.004 \\
\hline \multicolumn{3}{|c|}{ without dispersion } \\
\hline DTDA & 0.007 & 0.007 \\
$t$-NO & 0.007 & 0.007 \\
NNO & 0.003 & 0.003 \\
verdazyl & 0.012 & 0.012 \\
\hline \multicolumn{3}{|c}{ reference molecules } \\
\hline OPE & 0.008 & 0.008 \\
me-NO & 0.013 & 0.007 \\
TEMPO (cis $)$ & 0.002 & 0.002 \\
\hline \hline
\end{tabular}



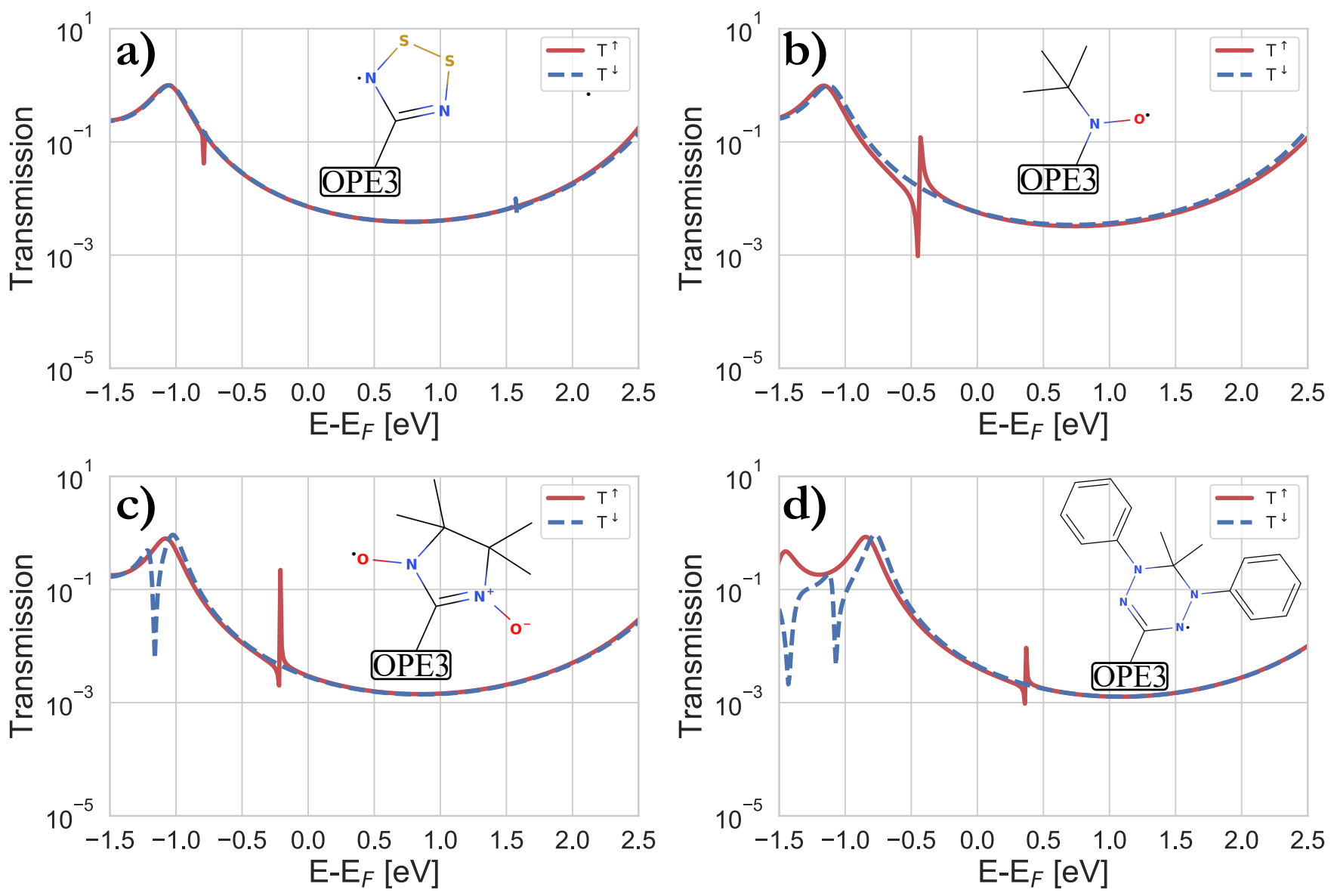

Figure 4: Calculated transmission functions for the OPE-based radicals under investigation. The energy was shifted w.r.t. the estimated Fermi energy $(-5 \mathrm{eV})$.

Dihedral angles defining the twist between radical substituents and OPE backbone vary between 26 and around 69 degrees, with $t$-NO-OPE being the most twisted and NNO-OPE coming second. For the $t$-NO radical, the preferred conformation also changes when omitting dispersion corrections, otherwise dispersion corrections play a minor role. For $t$-NO-OPE, the difference to a related compound discussed in Ref. [94] is striking: In that compound, the $t$-NO is attached to a a backbone with metarather than para-connection, leading to reduced steric interactions and much smaller dihedral angles $\left(9.8^{\circ} / 12.7^{\circ}\right)$, and accordingly to a substantial influence on spin-dependent electronic communication [94]. In contrast, for all four radicals studied here, as a consequence of the substituent twisting in the OPE wires the conjugation between the radical and the backbone $\pi$ systems is not enough for inducing a substantial spin delocalization onto the backbone (see Supporting Information) nor a

\footnotetext{
${ }^{1}$ The comparison is not strictly valid, as the angles in Ref. [94] were obtained with the BP86 exchange-correlation functional rather than B3LYP as in the present study. Yet, the typical variation between exchange-correlation functionals is much smaller than the difference discussed here.
} 
significant difference in $T^{\uparrow}$ and $T^{\downarrow}$ over a broad energy range (see Figure 4).

In the coherent tunneling regime, the electron going through regions of high unpaired spin density was shown to correlate with large differences between majority- (up) and minority- (down) spin electron transmission based on first-principles theoretical studies $[109,110,117]$. The small spin polarization found here is consistent with the local transmissions, which describe the conduction pathway of the electrons at certain energies, suggesting that, similar to TEMPO-OPE [11], the electrons at $E_{F}$ go through the OPE backbone and do not interact strongly with the spin-polarized radical part of the molecule (see Figure 5). For the verdazyl radical, a considerable difference for both spin channels occurs at around $-0.7 \mathrm{eV}$ below $E_{F}$, and for $t$-NO, a smaller but non-negligible difference around $-0.5 \mathrm{eV}$. In both cases, it might be possible to shift these features close to $E_{F}$ employing further chemical substituents [109].
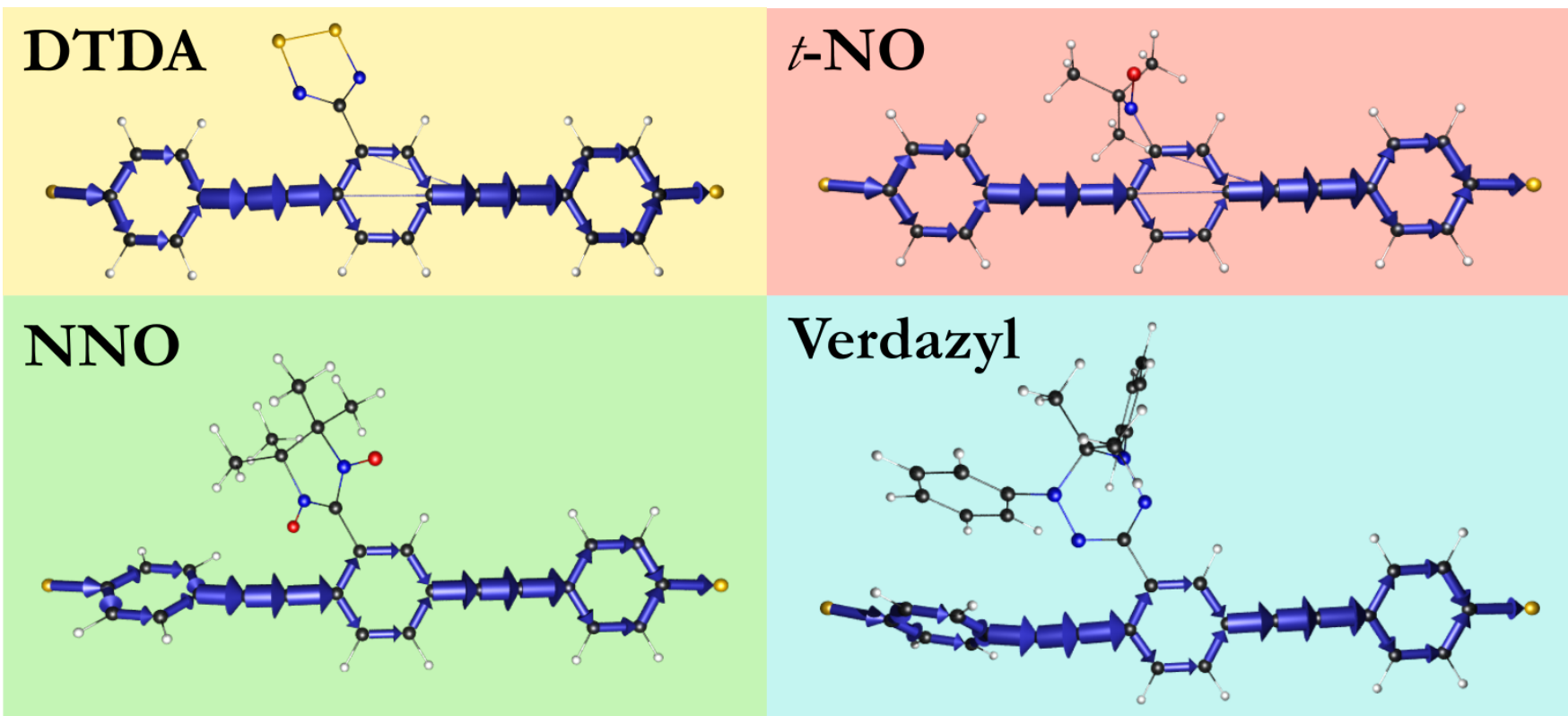

Figure 5: Calculated local transmissions for the four OPE-based radicals at the estimated $E_{F}$ of -5 $\mathrm{eV}$.

For all four radicals, Fano-resonance-like features occur in the transmission functions. These features can likely be attributed to destructive quantum interference [118-123], as discussed previously for para-connected benzene wires with substituents participating in the $\pi$-system $[109,124]$. The energetic location of these features can be understood in more detail by looking at the central-subsystem molecular orbitals (Figure 6), which represent the orbitals through which the electrons can tunnel: The features are located at the energies of the central-subsystem SOMOs (compare Figure 6 and Figure 4). The relatively high energy obtained for the SOMO of the verdazyl-substituted system 
is in line with an overall shift to higher energies of all orbitals, which is compatible with verdazyl being less electron-withdrawing than, e.g., NNO [125, 126]. The Fano-resonance-like features in the $|\downarrow\rangle$ transmissions will not be analyzed in detail since they are quite far from the Fermi energy (also, except for the DTDA radical, the energies of the $|\downarrow\rangle$-central-subsystem SOMOs are above the plotted energy range and can thus not be seen in the transmission). For the DTDA, the NNO, and the verdazyl radicals, these feature are very narrow. This is because the central-subsystem SOMO does not extend onto the OPE backbone and thus the coupling of the SOMO with the HOMO (which extends over the OPE backbone and plays the main part in mediating transport) is small. For the $t$-NO radical, parts of the central-subsystem SOMO extend onto the OPE backbone, which indicates a stronger coupling for this radical with the HOMO than for the other radicals, rationalizing why the Fano-resonance-like feature is more pronounced in the $t$-NO radical.
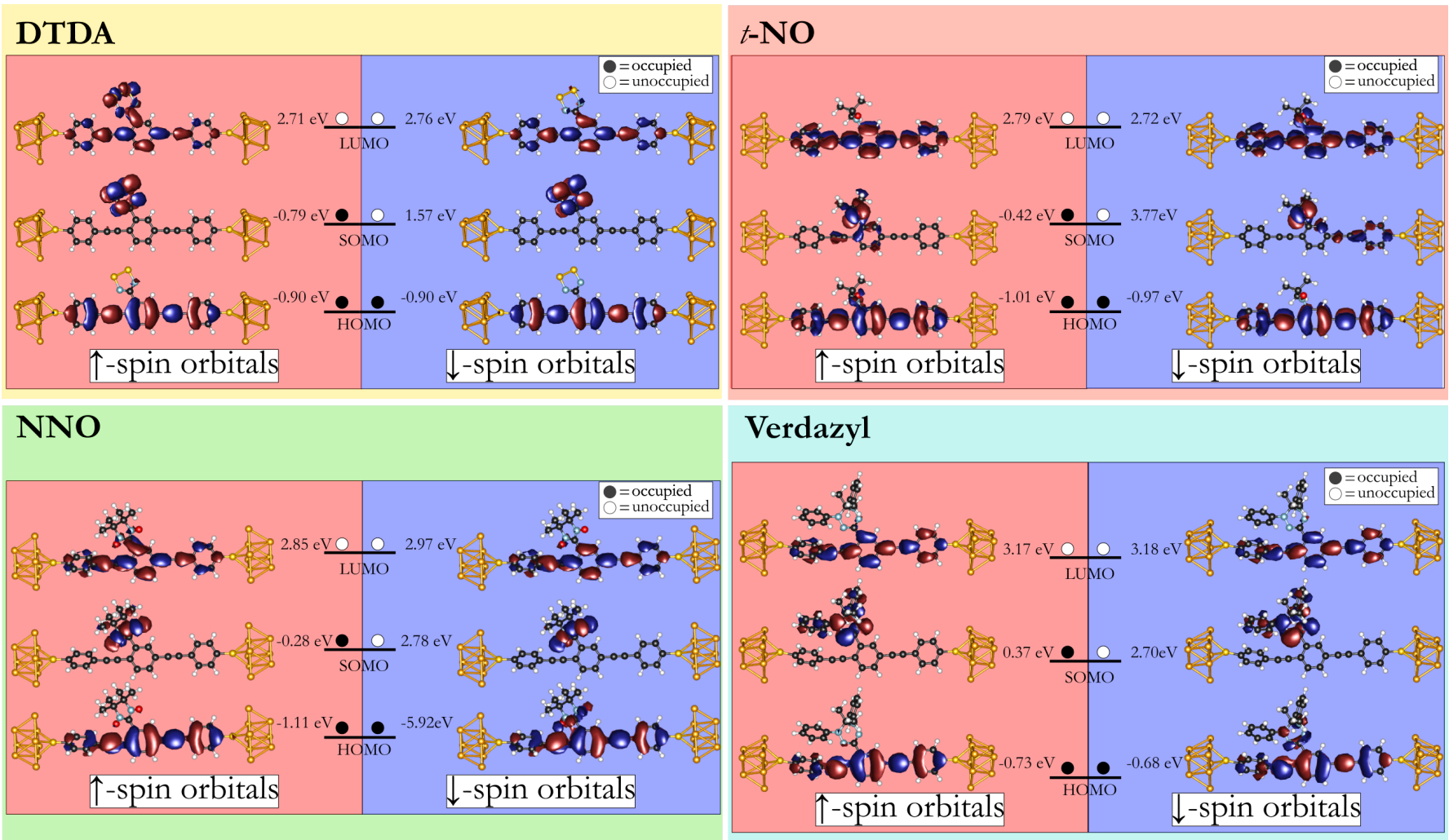

Figure 6: Calculated central-subsystem molecular orbitals for all four radicals (isosurface value: 0.025). The energies of the orbitals were shifted against the estimated Fermi energy of $-5 \mathrm{eV}$. For all radicals, the SOMO is mainly localized on the radical part of the molecule, while the HOMO and LUMO are mainly localized on the OPE backbone. 


\section{An artificial radical-OPE molecule with large spin polar- ization}

Based on the conclusions drawn above, one can suggest a structure for an organic radical with an OPE backbone which exhibits more strongly spin-dependent transport properties, e.g., an OPE molecule to which a methyl-nitroxide radical attached (see Figure 7). Since the methyl residue is small, the $\mathrm{N}-\mathrm{O}$ radical can be in plane with the OPE backbone, leading to good conjugation of radical and backbone $\pi$ systems. To fully enforce a completely planar structure, the results for this molecule are based on an optimized structure without taking into account dispersion interactions (however, even dispersion interactions do not lead to out-of plane rotation of the radical part here). This radical is rather artificial (most stable radicals have bulky residues or more delocalized spin densities to protect them from reactions such as oxidation, reduction or dimerization). Still, it is interesting to see how strong spin polarization can be for OPE wires with such an "ideal" substituent. As the calculated transmission functions for $|\uparrow\rangle$ and $|\downarrow\rangle$ electrons (see Figure 8) show, this strong conjugation of the methyl nitroxide with the OPE backbone leads to a significant difference between the transmissions for both spin channels over a broad energy range, as well as to a larger spin delocalization onto the backbone than for the tert-butyl-nitroxide-OPE (see Figure 9). A detailed analysis of the spinpolarized transmission as a function of the dihedral angle is shown in the Supporting Information. The stronger conjugation can also be observed in the central-subsystem molecular orbitals (see Figure 10). The HOMO and SOMO are both delocalized over the OPE-backbone and the radical part for the methyl nitroxide. For the tert-butyl nitroxide, this delocalization is much less pronounced.

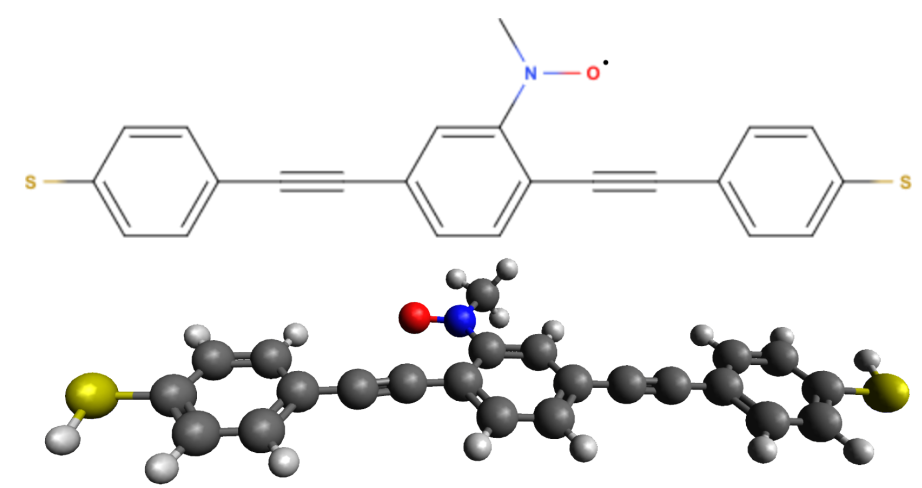

Figure 7: Lewis structure and optimized molecular structure (B3LYP/def2-TZVP) for the methylnitroxide-OPE molecule. Due to the small methyl residue, the complete structure of the molecule remains planar. 


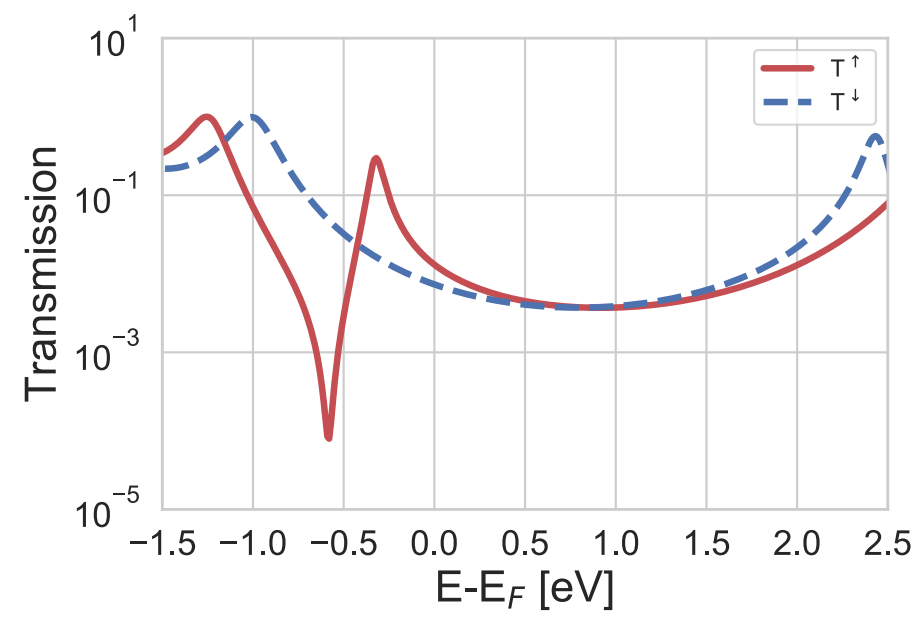

Figure 8: Calculated transmission function for the methyl-nitroxide-OPE molecule. The energy is shifted against the estimated $E_{F}$ of $-5 \mathrm{eV}$. A pronounced feature of the $\mathrm{SOMO}$ can be observed at about $-0.3 \mathrm{eV}$ and $|\uparrow\rangle$ and $|\downarrow\rangle$ electrons differ significantly over a wide range of energy.

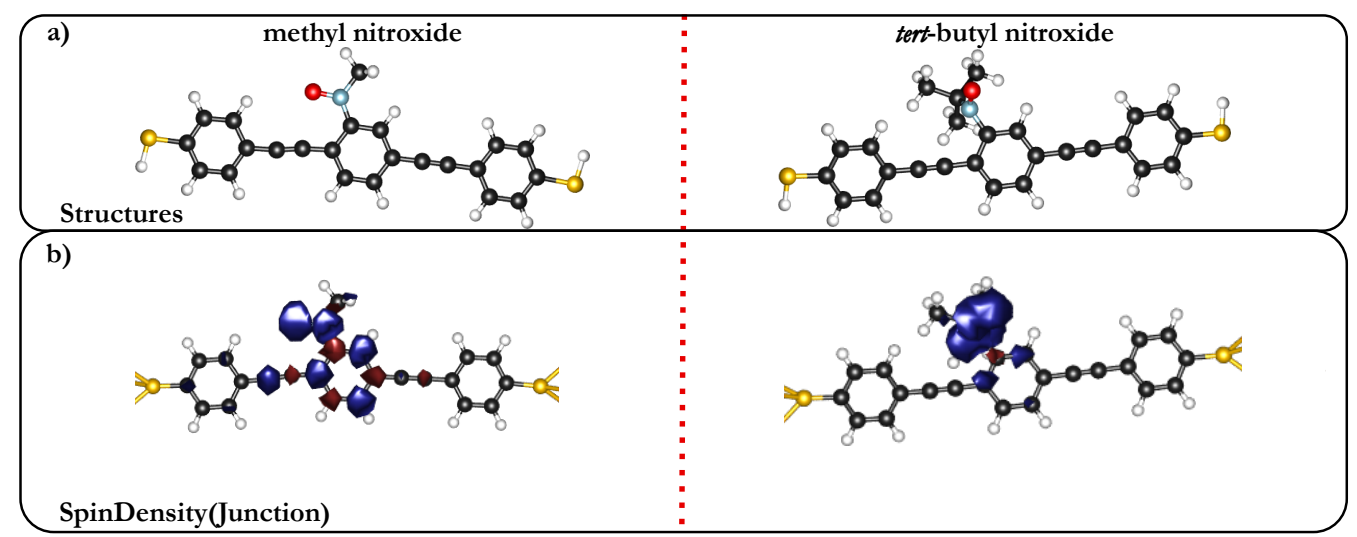

Figure 9: Optimized molecular structures and spin densities (isosurface value: 0.001) for the methyl nitroxide and tert-butyl nitroxide radicals. The spin density of the OPE-methyl-nitroxide expands onto the OPE backbone, while for the tert-butyl-nitroxide-OPE molecule, it is mainly localized on the radical. 
methyl nitroxide

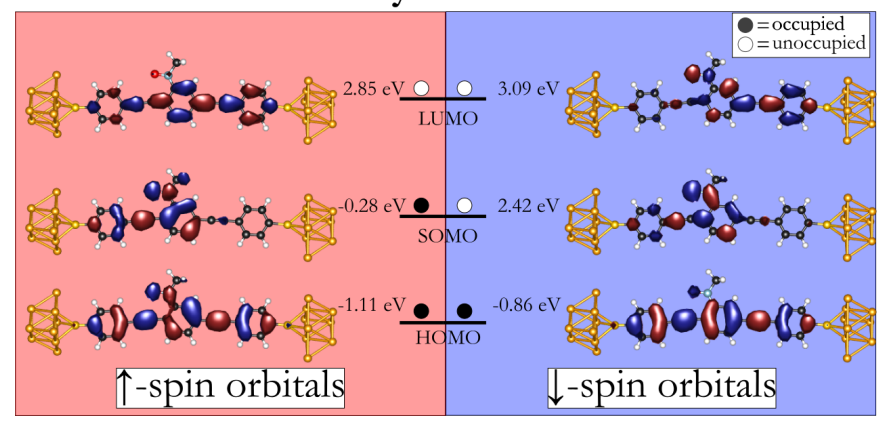

tert-butyl nitroxide

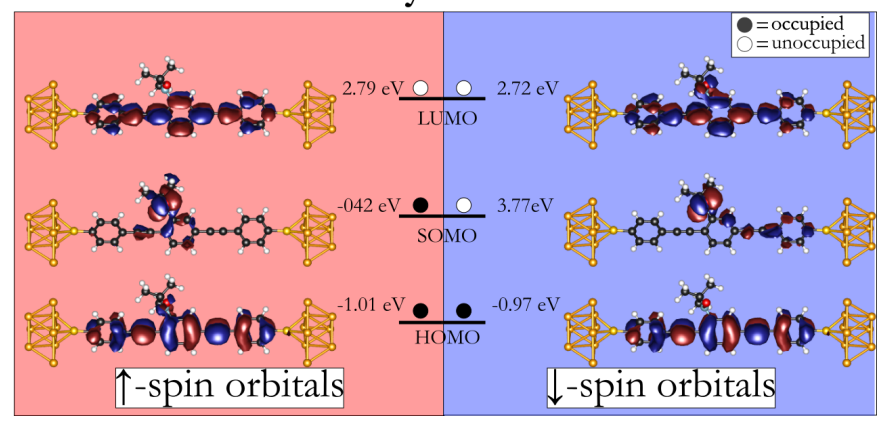

Figure 10: Calculated central-subsystem molecular orbitals for the methyl nitroxide and the tertbutyl nitroxide radicals (isosurface value: 0.025 ). The delocalization of the SOMO onto the OPE backbone and the delocalization of the HOMO on the radical part is much stronger for the methyl nitroxide radical than for the tert-butyl nitroxide radical. This is consistent with the much larger spin-dependence of the transport in the methyl nitroxide radical. The energies were shifted by the estimated Fermi energy $(-5 \mathrm{eV})$

\section{A mechanically flexible radical ligand: TEMPO-OPE}

In the "original" OPE radical, which inspired this study, a TEMPO radical is attached to the OPE backbone via an amide linker [11]. This results in a certain structural flexibility. Two isomers were identified to be stable and close in energy; the cis- and trans configuration with respect to the amide double bond. For the cis configuration, rotation of one of the OPE rings induced a significant decrease of the calculated transmission compared to the trans configuration. In both cases, DFT calculations indicate that the TEMPO radical does not induce a difference in the $|\uparrow\rangle /|\downarrow\rangle$ transmission in the Landauer regime (see Figure 11 and Ref. [11]). This leaves the open question how the large magnetoresistance of the molecule (as opposed to bare OPE) is caused. The structural flexibility of the radical substituent might open up additional possibilities of interactions, both with neighboring molecules and with the electrode surfaces, which might be related to the mechanism underlying magnetoresistance. Radicals close to metal surfaces can keep or loose their spin based on factors such as the environment (vacuum vs. solution) [73] (also see Ref. [72] for a review), which might lead to magnetic-field dependent properties if such interactions are present. Therefore, it is interesting to ask what the energy barrier is for transitions between the cis and trans configurations. A potential energy scan for a set of structures interpolating between the two, twisted stepwise around the amide bond with that bond dihedral fixed and all other degrees of freedom relaxed, followed by an optimization 
of the transition state, yields a barrier of $85 \mathrm{~kJ} / \mathrm{mol}$ (see Figure 12). This suggests that such transitions would be rather unlikely at the temperature of about $4 \mathrm{~K}$ at which the magnetoresistance measurements in Ref. [11] were done. To understand better whether one or the other is dominant in the experiment, or whether both are present, more detailed studies including intermolecular and molecule-electrode interactions will be needed. Furthermore, it has been shown that the presence of the metal surfaces can affect such energetic barriers substantially [127]. Such further studies will help understand whether structural flexibility, in addition to electronic considerations, can serve as a design principle for spin-dependent transport properties.
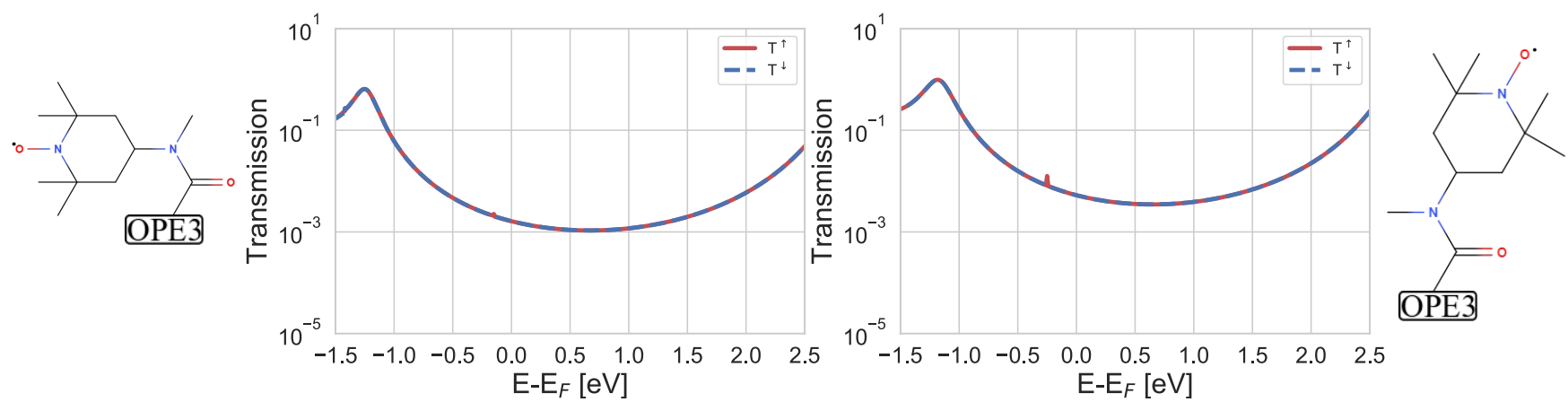

Figure 11: Calculated transmission functions of the TEMPO-OPE molecules (trans- and cis), as already discussed in Ref. [11]. The $|\uparrow\rangle /|\downarrow\rangle$ transmissions do not differ close to the estimated $E_{F}$ of $-5.0 \mathrm{eV}$. 

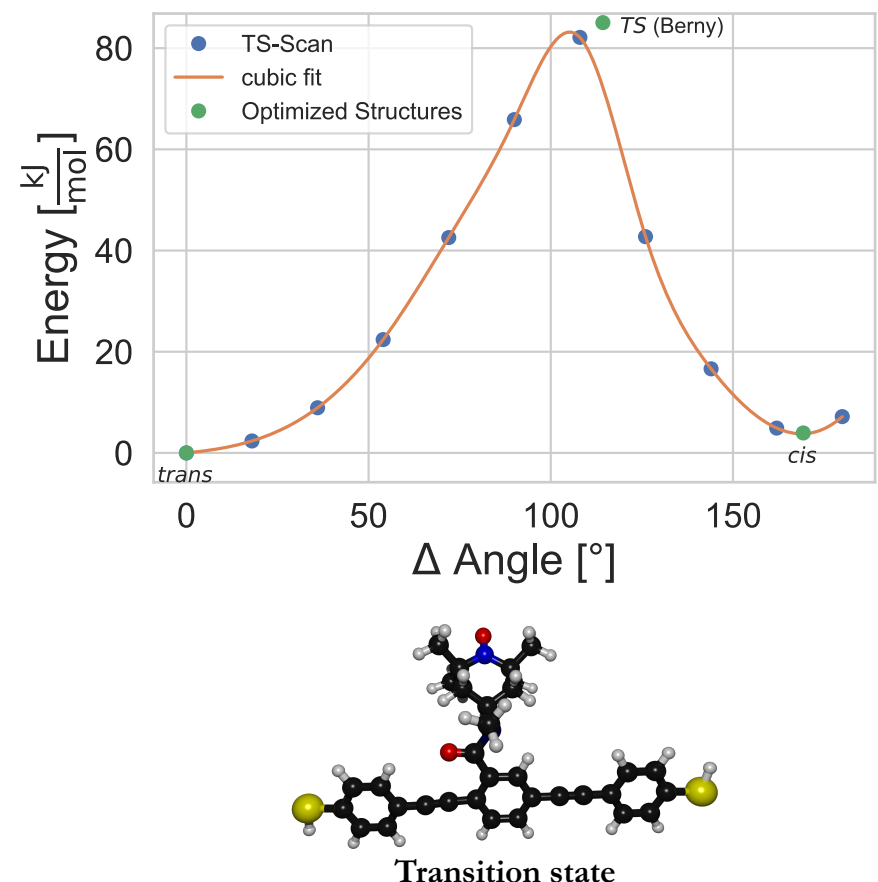

(Berny optimization)

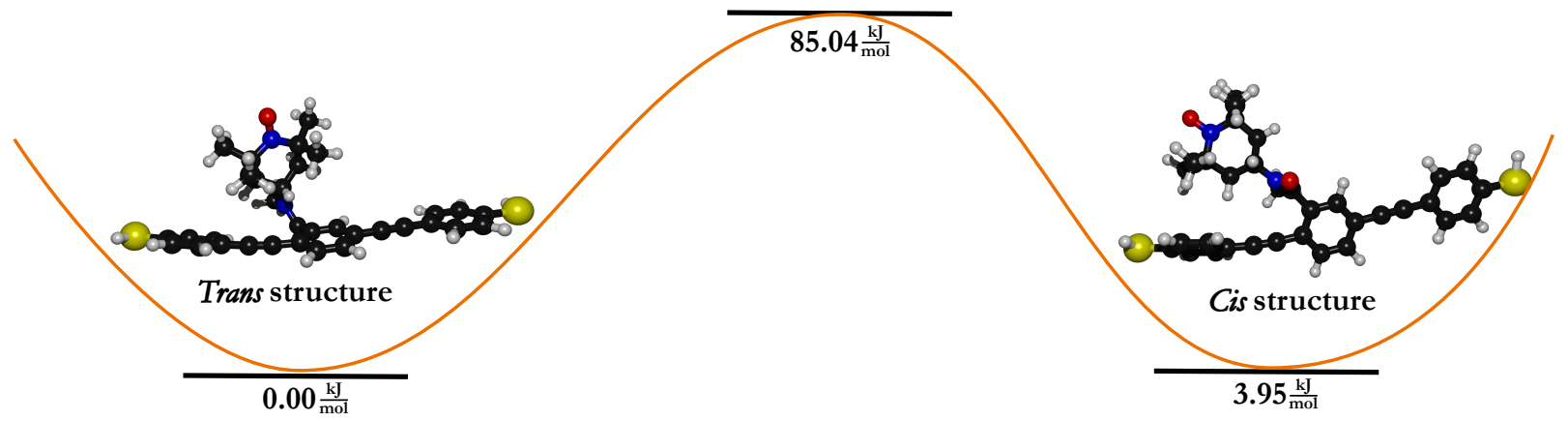

Figure 12: Calculated potential-energy surface (PES)for the transition from trans-TEMPO-OPE to cis-TEMPO-OPE. The energy is plotted against the dihedral angle formed by the nitrogen and carbon atoms of the amide bond and the nearest-neighbor carbon atoms of the OPE-backbone and the TEMPO radical. The blue dots represent the energy calculated from a PES scan, where the dihedral angle was changed successively and kept fixed during the following structure optimization. The green dots represent the optimized structures (cis, trans) and the structure of the transition state calculated with the "Berny" algorithm implemented in G09 [128,129]. The cis and trans structure only differ by about $4 \mathrm{~kJ} / \mathrm{mol}$. However, a rather high rotation barrier of $85 \mathrm{~kJ} / \mathrm{mol}$ makes a rotation unlikely under the experimental conditions $(T=4 \mathrm{~K})$ reported in Ref. [11]. 


\section{Conclusion}

We have studied the potential of different radical substituents to induce spin polarization in electron transmission through OPE wires by means of first-principles DFT calculations in the coherent tunneling regime. This is potentially relevant for measurements of shot noise, magnetoresistance, and Kondo properties.

All four stable substituents have their $\pi$ system twisted with respect to the OPE backbone, such that spin delocalization onto the OPE backbone, through which the electron is tunneling at small bias, is negligible. This results in negligible spin polarization of the transmission around the Fermi energy. For OPE-methyl-nitroxide $(t-\mathrm{NO})$, featuring the only substituent with spin density on the atom bonded to the backbone, non-negligible spin polarization at the Fermi energy might be achieved by shifting $\mathrm{MO}$ energies and thus transmission to higher energies by half an $\mathrm{eV}$, e.g. via further substituents. For verdazyl, this might also be possible if a shift of $0.7 \mathrm{eV}$ could be made. To achieve spin polarization over a broad energy range, however, it is necessary to have the substituent's $\pi$ system in plane with the OPE backbone's, e.g. by (in our case artificially) reducing steric interactions. A different approach for achieving larger spin polarization and possibly also more pronounced Kondo features would be to focus on meta-connected backbones, where it is easier to attach substituents with less steric interactions and thus with less twisting and better spin delocalization on the backbone (albeit at lower overall conductance).

The substituents' effects on overall transmission are minor when it comes to shifting up or down the energy scale. The main effect is a lowering of the overall transmission minimum, which is, at least for verdazyl, caused by dispersion interactions between the substituents and the backbone, twisting one of the outer phenyl rings. This is more pronounced the more "bulky" the substituent, i.e., in the order verdazyl $<\mathrm{NNO}<t$-NO $<$ DTDTA (where no such twisting is present). This is not unique to the substituent being a radical and could be considered as a general design tool for such compounds (after a careful assessment of conformational dynamics, given the easiness of rotation of the OPE rings [115]).

This twisting is also pronounced in TEMPO-OPE [11], yet here an additional property comes into play: The radical substituent is attached via an amide linker to the backbone, which opens up additional possibilities for interactions with other molecules or with the electrodes. We could show here that the ability of this ligand to change between the (nearly isoenergetic) cis and trans conformations should be restricted under the experimental conditions, at least for the isolated molecule. 
Given that TEMPO-OPE shows considerable single-molecule-magnetoresistance, such potential for interactions and flexibility, and how it could be affected by the environment [127] could be an additional interesting consideration for designing molecules with magnetism-dependent electron transport properties.

Finally, it would be highly interesting for future work to study the effect of these radical substituents on the spin-polarization of thiol-gold interfaces [67-71], which might alter the conductance properties beyond the purely molecule-centered effects studied here.

\section{Acknowledgments}

Financial support of this research by the German Research Foundation (DFG) via project HE 5675/61 is acknowledged. The authors thank the High-Performance Computing Center at University of Hamburg and the North-German Supercomputing Alliance (HLRN) for computational resources. We would like to thank Elke Scheer and Gautam Mitra for helpful discussions.

\section{$7 \quad$ Methodology}

All Kohn-Sham DFT calculations were performed with the program package GAUsSiAn09 [130]. The structures of the isolated molecules were optimized using the B3LYP [131-133] functional in combination with the def2-TZVP $[134,135]$ basis set. Dispersion interactions were taken into account by using the DFT-D3 [136] method in combination with Becke-Johnson [137] dampening (if not mentioned otherwise). Convergence criteria were $10^{-6}$ a.u. for the energy in the self-consistent-field algorithm and $10^{-3}$ a.u. for the gradient in the molecular structure optimizations.

The molecular junctions were constructed by placing the optimized isolated dithiol molecules between two nine-atomic gold clusters, having removed the hydrogen atoms of the thiol groups, mimicking hollow site adsorption on $\mathrm{Au}(111)$ surfaces, with sulfur-gold distances of $\mathrm{d}_{\mathrm{Au}-\mathrm{S}}=1.84 \AA$ from a previous DFT study [138]. The transmission functions were calculated with ARTAIOs [139], using the Fock and overlap matrix of a single-point calculation for the molecular junctions using the B3LYP functional in combination with the LANL2DZ [140-143] basis set, with the central (or scattering region) formed by the molecule only. Central-subsystem molecular orbitals were evaluated by cutting out the central-subsystem parts of the Fock matrix and solving the secular equations for that part only. They were analyzed rather than the full-junction MOs (which are often similar in shape but 
slightly shifted energetically) because peaks in the transmission are located at the energies of the central subsystem MOs.

Absolute conductance values will depend strongly on how and where a molecule contacts the electrodes. Molecular fluctuations therefore lead to conductance fluctuations up to several orders of magnitude during a break-junction measurement [7]. This implies that the information contained in a single measured conductance value is close to zero, but it is rather the statistical analysis of a large set of measured conductance values for a given junction that leads to meaningful and reproducible results (in particular the most likely conductance value). This is one of several factors which makes the first-principles prediction of absolute conductance values challenging (another being level alignment) [144]. However, qualitative trends in conductance and in transmission shapes when comparing different molecules tend to be independent of the details of electrode-molecule contacts [145-147], as long as the same contact configuration is used for each different molecule. Therefore, we compare the different radical-substituted OPE wires studied here employing one representative contact configuration as described above, keeping in mind that the resulting conductance properties should not be interpreted in quantitative, but only in qualitative terms.

\section{References}

[1] V. May, O. Kühn, Charge and Energy Transfer Dynamics in Molecular Systems; Wiley VCH: Weinheim, 2011.

[2] H. J. Wörner, et al. Struc. Dyn. 2017, 4, 061508.

[3] N. Zhang, S. R. Samanta, B. M. Rosen, V. Percec, Chem. Rev. 2014, 114, 5848-5958.

[4] P. Gehring, J. M. Thijssen, H. S. J. van der Zant, Nature Rev. Phys. 2019, 1, 381-396.

[5] G. C. Solomon, C. Herrmann, M. A. Ratner, Top. Curr. Chem. 2012, 313, 1-38.

[6] R. M. Metzger, Chem. Rev. 2015, 115, 5056-5115.

[7] J. C. Cuevas, E. Scheer, Molecular Electronics: An Introduction to Theory and Experiment; volume 1 of World Scientific Series in Nanotechnology and Nanoscience World Scientific: Singapore, 2nd ed.; 2017.

[8] Y. Komoto, S. Fujii, M. Iwane, M. Kiguchi, J. Mater. Chem. C 2016, 4, 8842-8858. 
[9] M. A. Ratner, Nature Nanotech. 2013, 8, 378-381.

[10] L. Sun, Y. A. Diaz-Fernandez, T. A. Gschneidtner, F. Westerlund, S. Lara-Avila, K. MothPoulsen, Chem. Soc. Rev. 2014, 43, 7378-7411.

[11] R. Hayakawa, M. A. Karimi, J. Wolf, T. Huhn, M. S. Zöllner, C. Herrmann, E. Scheer, Nano Lett. 2016, 16, 4960-4967.

[12] L. Mejia, N. Renaud, I. Franco, J. Phys. Chem. Lett. 2018, 9, 745-750.

[13] J. J. Parks, A. R. Champagne, T. A. Costi, W. W. Shum, A. N. Pasupathy, E. Neuscamman, S. Flores-Torres, P. S. Cornaglia, A. A. Aligia, C. A. Balseiro, G. K.-L. Chan, H. D. Abruña, D. C. Ralph, Science 2010, 328, 1370-1373.

[14] R. Frisenda, V. A. E. C. Janssen, F. C. Grozema, H. S. J. van der Zant, N. Renaud, Nature Chem. 2016, 8, 1099-1104.

[15] R. Frisenda, G. D. Harzmann, J. A. C. Gil, J. M. Thijssen, M. Mayor, H. S. J. van der Zant, Nano Lett. 2016, 16, 4733-4737.

[16] D. Stefani, K. J. Weiland, M. Skripnik, C. Hsu, M. L. Perrin, M. Mayor, Nano Lett. 2018, 9, $5981-5988$.

[17] S. Kawai, M. Koch, E. Gnecco, A. Sadeghi, R. Pawlak, T. Glatzel, J. Schwarz, S. Goedecker, S. Hecht, A. Baratoff, L. Grill, E. Meyer, Proc. Nat. Acad. Sci. 2014, 111, 3968-3972.

[18] L. Lafferentz, F. Ample, H. Yu, S. Hecht, C. Joachim, L. Grill, Science 2009, 323, 1193-1197.

[19] C. Bruot, L. Xiang, J. L. Palma, Y. Li, N. Tao, J. Am. Chem. Soc. 2015, 137, 13933-13937.

[20] V. Barone, A. Bencini, A. di Matteo, J. Am. Chem. Soc. 1997, 119, 10831-10837.

[21] V. Barone, A. Bencini, M. Cossi, A. D. Matteo, M. Mattesini, F. Totti, J. Am. Chem. Soc. 1998, 120, 7069-7078.

[22] D. Gatteschi, R. Sessoli, Angew. Chem. Int. Ed. 2003, 42, 268-297.

[23] D. Gatteschi, R. Sessoli, J. Villain, Molecular Nanomagnets; Oxford University Press: Oxford, 2005.

[24] L. Bogani, A. Vindigni, R. Sessoli, D. Gatteschi, J. Mater. Chem. 2008, 18, 4750-4758. 
[25] C. Climent, S. Vela, J. Jornet-Somoza, M. Deumal, Phys. Chem. Chem. Phys. 2019, 21, 1218412191.

[26] O. Kahn, Molecular Magnetism; Wiley VCH: New York, 1993.

[27] A. Bencini, D. HGatteschi, Electron Paramagnetic Resonance of Exchange Coupled Systems; Springer-Verlag: Berlin, 1990.

[28] S. Sanvito, Chem. Soc. Rev. 2011, 3336-3355.

[29] S. Roy, N. Shenvi, J. C. Tully, J. Phys. Chem. C 2011, 113, 16311-16320.

[30] C. Herrmann, Atomistic modeling for molecular electronics and spintronics: successes and challenges. In SPR Chemical Modelling, Vol. 14; J.-O. J. M. Springborg, (Ed.), RSC: London, 2018 .

[31] K. Yang, H. Chen, T. Pope, Y. Hu, L. Liu, D. Wang, L. Tao, W. Xiao, X. Fei, Y.-Y. Zhang, H.-G. Luo, S. Du, T. Xiang, W. A. Hofer, H.-J. Gao, Nature Commun. 2019, 10, 3599.

[32] L. L. Patera, S. Sokolov, J. Z. Low, L. M. Campos, L. Venkataraman, J. Repp, Angew. Chem. Int. Ed. 2019, 58, 11063-11067.

[33] S. Karan, C. Garcia, M. Karolak, D. Jacob, N. Lorente, R. Berndt, Nano Lett. 2018, 18, 88-93.

[34] F. Bejarano, I. J. Olavarria-Contreras, A. Droghetti, I. Rungger, A. Rudnev, D. Gutierrez, M. Mas-Torrent, J. Veciana, H. S. J. van der Zant, C. Rovira, E. Burzuri, N. Crivillers, J. Am. Chem. Soc. 2018, 140, 1691-1169.

[35] R. Frisenda, R. Gaudenzi, C. Franco, M. Mas-Torrent, C. Rovira, J. Veciana, I. Alcon, S. T. Bromley, E. Burzuri, H. S. J. van der Zant, Nano Lett. 2015, 15, 3109-3114.

[36] A. N. Pal, D. Li, S. Sarkar, S. Chakrabarti, A. Vilan, L. Kronik, A. Smogunov, O. Tal, Nature Commun. 2019, 10, 5565.

[37] M. Mohr, M. Gruber, A. Weismann, D. Jacob, P. Abufager, N. Lorente, R. Berndt, Phys. Rev. $B$ 2020, 101, 075414 .

[38] M. Noori, H. Sadeghi, C. J. Lambert, Nanoscale 2018, 10, 19220-19223.

[39] H. Gu, X. Zhang, H. Wei, Y. Huang, S. Wei, Z. Guo, Chem. Soc. Rev. 2013, 42, 5907-5943. 
[40] M. P. Bahlke, P. Wahl, L. Diekhöner, C. Herrmann, J. Appl. Phys. 2019, 125, 142910.

[41] A. Burtzlaff, A. Weismann, M. Brandbyge, R. Berndt, Phys. Rev. Lett. 2015, 114, 016602.

[42] R. Vardimon, M. Matt, P. Nielaba, J. C. Cuevas, O. Tal, Phys. Rev. B 2016, 93, 085439.

[43] A. Bencini, F. Totti, C. A. Daul, K. Doclo, P. Fantucci, V. Barone, Inorg. Chem. 1997, 36, 5022.

[44] C. Jacob, M. Reiher, Int. J. Quantum Chem. 2012, 112, 3661-3684.

[45] R. Naaman, Y. Paltiel, D. H. Waldeck, Nature Reviews 2019, 3, 250-260.

[46] K. Michaeli, N. Kantor-Uriel, R. Naaman, D. H. Waldeck, Chem. Soc. Rev. 2016, 45, 64786487.

[47] V. V. Maslyuk, R. Gutierrez, A. Dianat, V. Mujica, G. Cuniberti, J. Phys. Chem. Lett. 2018, 9, 5453-5459.

[48] M. S. Zöllner, S. Varela, E. Medina, V. Mujica, C. Herrmann, "Chiral-Induced Spin Selectivity: A Symmetry Analysis of Electronic Transmission", 2019 ChemRxiv, DOI: 10.26434/chemrxiv.8325248.v2.

[49] J. M. Abendroth, K. M. Cheung, D. M. Stemer, M. S. E. Hadri, C. Zhao, E. E. Fullerton, P. S. Weiss, J. Am. Chem. Soc. 2019, 141, 3863-3874.

[50] Z. Xie, T. Z. Markus, S. R. Cohen, Z. Vager, R. Gutierrez, R. Naaman, Nano Lett. 2011, 11, $4652-4655$

[51] T. J. Zwang, S. Hürlimann, M. G. Hill, J. K. Barton, J. Am. Chem. Soc. 2016, 138, 1555115554.

[52] B. Göhler, V. Hamelbeck, T. Z. Markus, M. Kettner, G. F. Hanne, Z. Vager, R. Naaman, H. Zacharias, Science 2011, 331, 894-897.

[53] D. Mishra, T. Z. Markus, R. Naaman, M. Kettner, B. Göhler, H. Zacharias, N. Friedman, M. Sheves, C. Fontanesi, Proc. Natl. Acad. Sci. 2013, 110, 14872-14876.

[54] A. C. Aragones, E. Medina, M. Ferrer-Huerta, N. Gimeno, M. Teixido, J. L. Palma, N. Tao, J. M. Ugalde, E. Giralt, I. Diez-Perez, V. Mujica, small 2017, 13, 1602519. 
[55] M. Kettner, B. Göhler, H. Zacharias, D. Mishra, V. Kiran, R. Naaman, C. Fontanesi, D. H. Waldeck, S. Sek, J. Pawlowski, J. Juhaniewicz, J. Phys. Chem. C 2015, 119, 14542-14547.

[56] M. Kettner, V. V. Maslyuk, D. Nürenberg, J. Seibel, R. Gutierrez, G. Cuniberti, K.-H. Ernst, H. Zacharias, J. Phys. Chem. Lett. 2018, 9, 2025-2030.

[57] V. Kiran, S. P. Mathew, S. R. Cohen, I. H. Delgado, J. Lacour, R. Naaman, Adv. Mater. 2016, $28,1957-1962$.

[58] S. Varela, V. Mujica, E. Medina, Chimia 2018, 72, 411-417.

[59] A.-M. Guo, Q. feng Sun, Phys Rev. Lett. 2012, 108, 218102.

[60] R. Gutierrez, E. Diaz, C. Gaul, T. Brumme, F. Dominguez-Adame, G. Cuniberti, J. Phys. Chem. C 2013, 117, 22276-22284.

[61] I. V. Krainov, J. Klier, A. P. Dmitriev, S. Klyatskaya, M. Ruben, W. Wernsdorfer, I. V. Gornyi, ACS Nano 2017, 11, 6868-6880.

[62] J.-J. Li, M.-L. Bai, Z.-B. Chen, X.-S. Zhou, Z. Shi, M. Zhang, S.-Y. Ding, S.-M. Hou, W. Schwarzacher, R. J. Nichols, B.-W. Mao, J. Am. Chem. Soc. 2015, 137, 5923-5929.

[63] A. C. Aragones, D. Aravena, F. J. Valverde-Munoz, J. A. Real, F. Sanz, I. Díez-Perez, E. Ruiz, J. Am. Chem. Soc. 2017, 139, 5768-5778.

[64] A. C. Aragonès, D. Aravena, J. I. Cerdá, Z. Acís-Castillo, H. Li, J. A. Real, F. Sanz, J. Hihath, E. Ruiz, I. Diez-Perez, Nano Lett. 2016, 16, 218-226.

[65] Z. Xie, S. Shi, F. Liu, D. L. Smith, P. P. Ruden*, C. D. Frisbie, ACS Nano 2016, 10, 8571-8577.

[66] S. Shi, Z. Xie, F. Liu, D. L. Smith, C. D. Frisbie, P. P. Rude, Phys. Rev. B 2017, 95, 155315.

[67] G. Rajaraman, A. Caneschi, D. Gatteschi, F. Totti, J. Mater. Chem. 2010, 20, 10747-10754.

[68] G. Rajaraman, A. Caneschi, D. Gatteschi, F. Totti, Phys. Chem. Chem. Phys. 2011, 13, 38863895.

[69] A. Caneschi, D. Gatteschi, F. Totti, Coord. Chem. Rev. 2015, 289-290, 357-378.

[70] R. Nabi, G. Rajaraman, Chem. Commun. 2019, 55, 8238-8241. 
[71] L. Puerta, H. J. Franco, J. Murgich, C. Gonzalez, Y. Simon-Manso, V. Mujica, J. Phys. Chem. A 2008, 112, 9771-9783.

[72] M. Mas-Torrent, N. Crivillers, C. Rovira, J. Veciana, Chem. Rev. 2011, 112, 2506-2527.

[73] J. Z. Low, G. Kladnik, L. L. Patera, S. Sokolov, G. Lovat, E. Kumarasamy, J. R. L. M. Campos, D. Cvetko, A. Morgante, L. Venkataraman, Nano Lett. 2019, 19, 2543-2548.

[74] H. Häkkinen, Nature Chemistry 2012, 4, 443-455.

[75] M. S. Inkpen, Z. Liu, H. Li, L. M. Campos, J. B. Neaton, L. Venkataraman, Nature Chem. 2019, 11, 351-358.

[76] G. Heimel, L. Romaner, E. Zojer, J.-L. Bredas, Acc. Chem. Res. 2008, 41, 721-729.

[77] V. Obersteiner, D. A. Egger, E. Zojer, J. Phys. Chem. C 2015, 119, 21198-21208.

[78] O. T. Hofmann, D. A. Egger, E. Zojer, Nano Lett. 2010, 10, 4369-4374.

[79] P. Lazić, M. Alaei, N. Atodiresei, V. Caciuc, R. Brako, S. Blügel, Phys. Rev. B 2010, 81, 045401.

[80] P. Janthon, F. Vines, J. Sirijaraensre, J. Limtrakul, F. Illas, J. Phys. Chem. C 2017, 121, 3970-3977.

[81] A. Patra, H. Peng, J. Sun, J. P. Perdew, Phys. Rev. B 2019, 100, 035442.

[82] Z.-F. Liu, D. A. Egger, S. Refaely-Abramson, L. Kronik, J. B. Neaton, J. Chem. Phys. 2017, 146, 092326.

[83] Z.-F. L. H. da JornadaSteven G. Louie, J. B. Neato, J. Chem. Theory Comput. 2019, 15, 4218-4227.

[84] K. S. Thygesen, A. Rubio, Phys. Rev. Lett. 2009, 102, 046802.

[85] J. B. Neaton, M. S. Hybertsen, S. G. Louie, Phys. Rev. Lett. 2006, 97, 216405.

[86] X. Xiao, L. A. Nagahara, A. M. Rawlett, N. Tao, J. Am. Chem. Soc. 2005, 127, 9235-9240.

[87] Y. Karzazi, J. Cornil, J. L. Bredas, Nanotechnology 2003, 14, 165-171. 
[88] H. Lissau, R. Frisenda, S. T. Olsen, M. Jevric, C. R. Parker, A. Kadziola, T. Hansen, H. S. J. van der Zant, M. B. Nielsen, K. V. Mikkelsen, Nature Comm. 2015, 6, 10233.

[89] Z. Wei, et al. Adv. Funct. Mater. 2015, 25, 1700-1708.

[90] C. Traina, L. Norela, M. Baumgarten, Coord. Chem. Rev. 2009, 253, 2342-2351.

[91] I. Ratera, J. Veciana, Chemical Society Reviews 2012, 41, 303-349.

[92] J. M. Rawson, A. Alberola, A. Whalley, Journal of Materials Chemistry 2006, 16, 2560-2575.

[93] J. M. Rawson, J. J. Hayward, .1: Stable Chalcogen Radicals. In Handbook of Chalcogen Chemistry; Royal Society of Chemistry: London, 2013.

[94] T. Steenbock, D. A. Shultz, M. L. Kirk, C. Herrmann, J. Phys. Chem. A 2017, 121, 216-225.

[95] C. Herrmann, J. Elmisz, Chem. Commun. 2013, 49, 10456-10458.

[96] M. L. Kirk, D. A. Shultz, E. C. Depperman, C. L. Brannen, J. Am. Chem. Soc. 2007, 129, 1937-1943.

[97] M. L. Kirk, D. A. Shultz, D. E. Stasiw, G. F. Lewis, G. Wang, C. L. Brannen, R. D. Sommer, P. D. Boyle, Journal of the American Chemical Society 2013, 135, 17144-17154.

[98] G. Blondin, J. J. Girerd, Chem. Rev. 1990, 90, 1359-1376.

[99] F. Tuczek, E. I. Solomon, J. Am. Chem. Soc. 1994, 116, 6916-6924.

[100] S. Shil, P. Sarbadhikary, A. Misra, RSC Adv. 2016, 6, 99467-99474.

[101] P. Sarbadhikary, S. Shil, A. Misra, Phys. Chem. Chem. Phys. 2018, 20, 9364-9375.

[102] J. Proppe, C. Herrmann, J. Comput. Chem. 2015, 36, 201-209.

[103] C. Herrmann, J. Phys. Chem. A 2019, 123, 10205-10223.

[104] E.-D. Fung, D. Gelbwaser, J. Taylor, J. Low, J. Xia, I. Davydenko, L. M. Campos, S. Marder, U. Peskin, L. Venkataraman, Nano Lett. 2019, 19, 2555-2561.

[105] Q. Lu, K. Liu, H. Zhang, Z. Du, X. Wang, F. Wang, ACS Nano 2009, 3, 3861-3868.

[106] Q. Lu, C. Yao, X. Wang, F. Wang, J. Phys. Chem. C 2012, 116, 17853-17861. 
[107] C. Caroli, R. Combescot, P. Nozieres, D. Saint-James, J. Phys. C: Solid State Phys. 1971, 4, 916-929.

[108] C. Herrmann, G. C. Solomon, J. E. Subotnik, V. Mujica, M. A. Ratner, J. Chem. Phys 2010, $132,024103$.

[109] C. Herrmann, G. C. Solomon, M. A. Ratner, J. Am. Chem. Soc. 2010, 132, 3682-3684.

[110] C. Herrmann, G. C. Solomon, M. A. Ratner, J. Chem. Phys. 2011, 134, 224306.

[111] T. Sugawara, M. Minamoto, M. M. Matsushita, P. Nickels, S. Komiyama, Phys. Rev. B 2008, 77,235316 .

[112] T. Sugawara, M. M. Matsushita, Journal of Materials Chemistry 2009, 19, 1738-1753.

[113] S. Krause, L. Berbil-Bautista, G. Herzog, M. Bode, R. Wiesendanger, Science 2007, 317, $1537-1540$

[114] N. Lorente, J.-P. Gauyacq, Phys. Rev. Lett. 2009, 103, 176601.

[115] J. M. Seminario, A. G. Zacarias, J. M. Tour, J. Am. Chem. Soc. 1998, 120, 3970-3974.

[116] L. Venkataraman, J. E. Klare, C. Nuckolls, M. S. Hybertsen, M. L. Steigerwald, Nature 2006, 442, 904-907.

[117] M. Smeu, G. A. DiLabio, J. Phys. Chem. C 2010, 114, 17874-17879.

[118] A. Borges, J. Xia, S. H. Liu, L. Venkataraman, G. C. Solomon, Nano Lett. 2017, 17, 4436-4442.

[119] M. Carlotti, S. Soni, X. Qiu, E. Sauter, M. Zharnikov, R. C. Chiechi, Nanoscale Adv. 2019, -.

[120] C. J. Lambert, S.-X. Liu, Chem. Eur. J. 2018, 24, 4193.

[121] T. Markussen, R. Stadler, K. S. Thygesen, Nano Lett. 2010, 10, 4260-4265.

[122] S.-H. Ke, W. Yang, H. U. Baranger, Nano Lett. 2008, 8, 3257-3261.

[123] Y. Tsuji, E. Estrada, R. Movassagh, R. Hoffmann, Chem. Rev. 2018, 118, 4887-4911.

[124] H. Schlicke, C. Herrmann, ChemPhysChem 2014, 15, 4011-4018.

[125] V. Chemistruck, D. Chambers, D. J. R. Brook, J. Org. Chem. 2009, 74, 1850-1857. 
[126] E. Coronado, C. Gimenez-Saiz, M. Nicolas, F. M. Romero, E. Rusanov, H. Stoeckli-Evans, New J. Chem. 2003, 27, 490-497.

[127] A. Schlimm, R. Löw, T. Rusch, F. Röhricht, T. Strunskus, T. Tellkamp, F. Sönnichsen, U. Manthe, O. Magnussen, F. Tuczek, R. Herge, Angew. Chem. Int. Ed. 2019, 58, 6574-6578.

[128] C. Peng, H. B. Schlegel, Isr. J. Chem. 1993, 33, 449-454.

[129] C. Peng, P. Y. Ayala, H. B. Schlegel, M. J. Frisch, J. Comp. Chem. 1996, 17, 49-56.

[130] M. J. Frisch, et al. "Gaussian 09 Revision A.1", Gaussian Inc. Wallingford CT 2009.

[131] A. D. Becke, Phys. Rev. A 1988, 38, 3098-3100.

[132] C. Lee, W. Yang, R. G. Parr, Phys. Rev. B 1988, 37, 785-789.

[133] A. D. Becke, J. Chem. Phys. 1993, 98, 5648-5652.

[134] A. Schäfer, C. Huber, R. Ahlrichs, J. Chem. Phys. 1994, 100, 5829-5835.

[135] F. Weigend, R. Ahlrichs, Phys. Chem. Chem. Phys. 2005, 7, 3297-3305.

[136] S. Grimme, J. Antony, S. Ehrlich, H. Krieg, J. Chem. Phys. 2010, 132, 154104.

[137] S. Grimme, S. Ehrlich, L. Goerigk, J. Comput. Chem. 2011, 32, 1456-1465.

[138] A. Bilić, J. R. Reimers, N. S. Hush, J. Chem. Phys. 2005, 122, 094708.

[139] M. Deffner, L. Groß, T. Steenbock, B. A. Voigt, M. S. Zöllner, G. C. Solomon, C. Herrmann, 2008-2020 ArTAios - a postprocessing framework for electronic structure calculations, https://www.chemie.unihamburg.de/institute/ac/arbeitsgruppen/herrmann/software/artaios.html.

[140] T. H. Dunning Jr., P. J. Hay, Modern Theoretical Chemistry. In , Vol. 3; H. F. Schaefer III, (Ed.), New York, 1977.

[141] P. J. Hay, W. R. Wadt, J. Chem. Phys. 1985, 82, 270.

[142] W. R. Wadt, P. J. Hay, J. Chem. Phys. 1985, 82, 284.

[143] P. J. Hay, W. R. Wadt, J. Chem. Phys. 1985, 82, 299. 
[144] F. Evers, R. Korytar, S. Tewari, J. M. van Ruitenbeek, 2019, arXiv:1906.10449v1.

[145] S.-H. Ke, H. U. Baranger, W. Yang, Phys. Rev. B 2004, 70, 085410.

[146] S.-H. Ke, H. U. Baranger, W. Yang, J. Chem. Phys. 2005, 123, 114701.

[147] F. Chen, X. Li, J. Hihath, Z. Huang, N. Tao, J. Am. Chem. Soc. 2006, 128, 15874-15881. 